\title{
CME-Driven and Flare-Ignited Fast Magnetosonic Waves Successively Detected in a Solar Eruption
}

\author{
Xinping Zhou ${ }^{1,2,3,4}$ (D) \\ Yuandeng Shen ${ }^{1,2,3,4}$ (D) Jiangtao $\mathrm{Su}^{3,4}$. \\ Zehao Tang $^{1,4}$ (D) Chengrui Zhou ${ }^{1,4}$. \\ Yadan $\operatorname{Duan}^{1,4}$ (D) Song Tan ${ }^{1,4}$ \\ (C) Springer $\bullet \bullet \bullet$
}

\begin{abstract}
We present $S D O /$ AIA observation of three types of fast-mode propagating magnetosonic waves in a GOES C3.0 flare on 2013 April 23, which was accompanied by a prominence eruption and a broad coronal mass ejection (CME). During the fast rising phase of the prominence, a large-scale domeshaped extreme ultraviolet (EUV) wave firstly formed ahead of the CME bubble and propagated at a speed of about $430 \mathrm{~km} \mathrm{~s}^{-1}$ in the CME's lateral direction. One can identify the separation process of the EUV wave from the CME bubble. The reflection effect of the on-disk counterpart of this EUV wave was also observed when it interacted with a remote active region. Six minutes after the first appearance of the EUV wave, a large-scale quasi-periodic EUV train with a period of about 120 seconds appeared inside the CME bubble, which emanated from the flare epicenter and propagated outward at an average speed up to $1100 \mathrm{~km} \mathrm{~s}^{-1}$. In addition, another narrow quasi-periodic EUV wave train was observed along a closed-loop system connecting two adjacent active regions, which also emanated from the flare epicenter, propagated at a speed of about 475 $\mathrm{km} \mathrm{s}^{-1}$ and with a period of about 110 seconds. We propose that all the observed waves are fast-mode magnetosonic waves, in which the large-scale dome-shaped EUV wave ahead of the CME bubble was driven by the expansion of the CME bubble, while the large-scale quasi-periodic EUV train within the CME bubble
\end{abstract}

\footnotetext{
$\bowtie$ Y.D. Shen

ydshen@ynao.ac.cn

1 Yunnan Observatories, Chinese Academy of Sciences, Kunming 650011, China

2 State Key Laboratory of Space Weather, Chinese Academy of Sciences, Beijing 100190, China

3 Key Laboratory of Solar Activity, National Astronomical Observatories, Chinese Academy of Sciences, Beijing 100012, China

4 University of Chinese Academy of Sciences, Beijing 10049,China
} 
and the narrow quasi-periodic EUV wave train along the closed-loop system were excited by the intermittent energy-releasing process in the flare. Coronal seismology application and energy carried by the waves are also estimated based on the measured wave parameters.

Keywords: Waves, magnetohydrodynamic; Magnetic fields, Corona; Coronal seismology

\section{Introduction}

Large-scale magnetohydrodynamic (MHD) waves in the solar atmosphere such as umbra and penumbra of sunspots running waves(e.g., Zhou et al., 2017; Zhou and Liang, 2017), chromospheric Moreton waves, and coronal extreme ultraviolet (EUV) waves have been investigated for many years (e.g., Moreton and Ramsey, 1960; Shen and Liu, 2012c; Krause et al., 2018; Wang et al., 2020). The measured physical parameters of MHD waves are crucial for diagnosing their physical nature, excitation mechanism, and energy transport in the solar atmosphere. Large-scale EUV disturbances are typically interpreted as fast-mode magnetosonic waves, which can propagate globally and generally interact with various coronal structures. For example, they can cause the oscillation of remote filaments and coronal loops (Schrijver, Aschwanden, and Title, 2002; Wills-Davey and Thompson, 1999; Aschwanden et al., 1999; Tripathi, Isobe, and Jain, 2009; Liu et al., 2013; Shen et al., 2014; Kumar et al., 2013; Shen et al., 2019). The wave nature can be evidenced through reflection, refraction, and transmission effects when EUV waves interacted with remote coronal holes (Gopalswamy et al., 2009; Li et al., 2012; Olmedo et al., 2012; Liu et al., 2018; Hu et al., 2019; Liu et al., 2019), active regions (Wills-Davey and Thompson (1999), Ofman and Thompson (2002),Ofman (2007),Kumar and Innes (2013), Shen et al. (2013b),Kumar and Manoharan (2013)). Especially, the oscillation phenomena of the filaments or prominences after impingement are very suitable for local seismology, which is a good technique for estimating the magnetic field strength of the filament and the surrounding coronal environment. EUV waves also carry critical information that can be used to diagnose some important physical parameters of the corona such as the magnetic field strength, which is an area yet to be fully exploited (e.g., Long, DeLuca, and Gallagher (2011); Shen et al. (2019); Uchida (1970); Nakariakov and Verwichte (2005); Zhou and Liang (2017); Miao et al. (2021)).

Large-scale coronal EUV waves can propagate across a large fraction the solar disk with a typical speed of 200-400 $\mathrm{km} \mathrm{s}^{-1}$ (Thompson and Myers, 2009), which were firstly observed by the EUV Imaging Telescope (EIT; Delaboudinière et al. (1995)) onboard the Solar and Heliospheric Observatory spacecraft (SOHO; (Moses et al., 1997; Thompson et al., 1998)). Over the past two decades, though a large number of wave events have been analyzed with various observations, there are still different opinions regarding their driving mechanism and physical nature. Large-scale EUV waves were initially believed to be driven by the flare pressure pulses as the chromospheric Moreton waves Uchida (1968) (e.g., Khan and Aurass (2002); Hudson et al. (2003); Warmuth et al. (2004)). 
After the launch of the Solar Dynamics Observatory (SDO;Pesnell, Thompson, and Chamberlin (2012)) in 2010, the high temporal and high spatial resolution observations taken by the Atmospheric Imaging Assembly (AIA; Lemen et al. (2012)) pushed forward the research for EUV waves to a new climax. More and more observational evidence has shown that large-scale EUV waves are closely associated with CMEs rather than flare pressure pulses (Chen (2006); Shen and Liu (2012b,a); Xue et al. (2013); Zheng et al. (2012a); Cliver et al. (2005); Long et al. (2017); Shen et al. (2017a)). Regarding the physical nature, EUV waves are generally recognized as fast-mode magnetosonic waves driven by CMEs. Some observations demonstrated the simultaneous existing of two types of waves (e.g., Zhukov and Auchère, 2004; Chen and Wu, 2011; Shen and Liu, 2012c; Shen et al., 2013b, 2014; Liu et al., 2012; Guo, Ding, and Chen, 2015; Chandra et al., 2018; Kumar and Innes, 2013), as what have been predicted in Chen et al. (2002); Chen, Fang, and Shibata (2005), i.e., a fast true wave followed by a slow pseudo one. It is worth pointing out that CMEs are not a necessary requirement for producing large-scale EUV waves, although they have been generally accepted as the driver of EUV waves. Some recent observations have shown that large-scale EUV waves can also be driven by various non-CMEassociation solar eruptions, such as coronal jets (Shen et al., 2018b,c,d; Zheng et al., 2013), mini-filament eruptions (Wang et al., 2020), expanding coronal loops (Su et al., 2015; Shen et al., 2017a, 2018b,c), and newly formed sigmoidal loops (Zheng et al., 2012b). Recently, large-scale quasi-periodic EUV waves with periods of a few minutes have been detected, which can propagate simultaneously along and across magnetic field lines and cause the oscillations of filaments and coronal loops(Liu et al., 2012; Shen et al., 2019). Shen et al. (2019) proposed that the excitation of the large-scale quasi-periodic EUV wave train was possibly due to the period opening of the unwinding helical threads of the associated erupting filament.

A new important finding of the $S D O$ was the discovery of the so-called quasiperiodic fast propagating (QFP) magnetosonic wave trains (Liu et al., 2011). QFP wave trains are composed of multiple coherent narrow arc-shaped wavefronts, propagating along open or closed coronal loops with high speeds up to $2000 \mathrm{~km} \mathrm{~s}^{-1}$. Since one or more periods of a QFP wave train are generally consistent with that of the accompanying flares, they are believed to be excited by the periodic energy release process in flares (e.g., Liu et al. (2011),Shen and Liu (2012b),Shen et al. (2013a), Yuan et al. (2013),Kumar and Manoharan (2013),Kumar, Nakariakov, and Cho (2017),Shen et al. (2018a), Miao et al. (2021)). Besides, the leakage p-model oscillations from photospheric to the corona (e.g., Shen and Liu, 2012b; Qu, Jiang, and Chen, 2017), the dispersive evolution (e.g., Yuan et al., 2013; Nisticò, Pascoe, and Nakariakov, 2014; Shen et al., 2018a,c) and even CMEs (Miao et al., 2019) are also considered as the possible exciter for QFP wave trains. According to Liu and Ofman (2014), QFP wave trains with a series of arc-shaped wavefronts at speeds of $500-2200 \mathrm{~km} \mathrm{~s}^{-1}$ propagate upward along funnel- or conic-like coronal loops within a limited angular sector (say, $10^{\circ}-60^{\circ}$ ), which indicate that QFP wave trains are trapped in low Alfvén structures that serve as waveguides (Roberts, Edwin, and Benz, 1983, 1984). The lifetimes (periods) of QFP wave trains range from several minutes to 
more than one hour (25-400 seconds). So far, the direct observations of QFP wave trains are still relatively scarce. However, according to Liu et al. (2016), at least one-third of events associated with large-scale EUV waves, flares, and CMEs are accompanied by QFP wave trains. The lack of a detailed case to analyze QFP wave trains is probable because the QFP waves can only lead to a subtle intensity fluctuation (say, $1 \%-5 \%$ ) in EUV images. A few two- and threedimensional MHD simulation works were also performed to study the QFP wave trains (Ofman et al., 2011; Pascoe, Nakariakov, and Kupriyanova, 2013; Takasao and Shibata, 2016), and some of them can perfectly reproduce QFP wave trains which have similar amplitude, wavelength, and propagation speeds with real observations (e.g., Ofman et al., 2011).

In this paper, we report a rare observations of simultaneous detection of three types of fast-mode magnetosonic waves in a prominence eruption event on 2013 April 23. The eruption was accompanied by GOES C3.0 flare and a broad CME. During the eruption, we observed a large-scale EUV wave ahead of the CME front, a large-scale quasi-periodic EUV wave train inside the CME bubble, and a narrow quasi-periodic EUV wave train trapped by a closed-loop system. The co-existence of the three types of wave phenomena offers an excellent opportunity to understand their driving mechanisms, propagation properties, and the surrounding coronal conditions.

\section{Observations and Data Analysis}

AIA onboard the $S D O$ provides full-disk $(4096 \times 4096$ pixels $)$ images of the corona and transition region at seven narrow EUV channels, at a high pixel resolution of $0 . " 6$, a temporal cadence of 12 seconds, and a wide field of view (FOV) of $1.3 R_{\odot}$. The EUV emission lines cover a wide range of temperature from $1 \mathrm{MK}$ to $20 \mathrm{MK}$, which thus offers a good opportunity for us to study the kinematics and thermal feature of various fast-propagating wave phenomena in the corona. Here we mainly use the images at $171 \AA$ (Fe IX; characteristic temperature: $0.6 \times 10^{6}$ $\mathrm{K}), 193 \AA$ (Fe XII,XXIV; characteristic temperature: $1.6 \times 10^{6} \mathrm{~K}, 2 \times 10^{7} \mathrm{~K}$ ) and $304 \AA$ (He II; characteristic temperature: $0.5 \times 10^{5} \mathrm{~K}$ ). All of the AIA images used here are calibrated and differentially rotated to a reference time (18:00:00) with the standard procedure "aia_prep.pro" available in the SolarSoftWare (SSW) package. Since the exposure times of the AIA images vary in response to the increase of flare emission, we normalized it by dividing the exposure time recorded in the fits header of each image. The radio spectrographs provided by the Radio and Plasma WAVE Experiment (WAVES; Bougeret et al. (1995)) aboard Wind spacecraft are used to analyze the associated interplanetary radio signal, which sweeps the frequency range $0.02-1.04 \mathrm{MHz}$ (RAD1) and 1.075-13.825 $\mathrm{MHz}$ (RAD2) every minute. We also used the Potential Field Source Surface (PFSS; Schrijver and De Rosa (2003)) model to show the magnetic loop systems of interest. In addition, Large Angle and Spectrometric COronagraph (LASCO; Brueckner et al. (1995)) images are used to portray the associated CME caused by the eruption of prominence. Geostationary Operational Environmental Satellite (GOES) 0.5-4 $\AA$ flux is used to analyze the periodicity of the accompanying 
C3.0 flare. To highlight the moving features, for example, the wavefronts, we utilize running-difference and base-difference images to study the kinematics and propagating characteristics of the EUV waves, in which the time difference $(\Delta t)$ for running difference images and reference time for base-difference images are 12 seconds and 18:00:00 UT, respectively. To show the kinematic features of the propagating disturbance, we estimated its speed using time-distance stack plots (TDSPs) created by stacking a one-dimensional intensity profile along the center of propagating path of interest, in which the gradient of a oblique stripe represents the propagating disturbance speed. The technique of wavelet analysis (Torrence and Compo (1998)) is used to investigate the periodicity of the EUV wave trains and flare.

\section{Result}

The event occurred on 2013 April 23 at about 18:00 UT in NOAA active region AR11723 that was close to the west limb of the solar disk from the Earth's angle of view. Such a limb event is in favor of investigating the relationship between the associated CME and EUV waves. An overview of the initial magnetic topology of the eruption source region before the event is shown in Figure 1 (a), in which some extrapolated magnetic field lines are overlaid. We focus on two groups of loop systems: the one is a closed trans-equatorial loop system L1 that connected the active regions of AR11723 and AR11726 in opposite hemispheres, the other one L2 is an active region loop whose two footpoints were rooted in opposite magnetic polarity regions of AR11726. This event started with the rising and eruption of a loop-like prominence, and was associated with a broad CME with an average speed of $403 \mathrm{~km} \mathrm{~s}^{-1}{ }^{1}$ (see Figure 1 (e)), a GOES soft X-ray C3.0 flare, a type III radio burst, and various EUV waves. The start, peak, and end times of the flare were at about 18:10 UT, 18:33 UT, and 18:45 UT, respectively. In this paper, we mainly focus on the driving mechanisms of the accompanying EUV waves, including a large-scale EUV wave traveling ahead of the CME bubble, a quasi-periodic large-scale EUV wave train running upwardly within the CME bubble (i.e., behind the CME front), and a narrow quasi-periodic EUV wave train propagating along the closed-loop system L1. In the following subsections, we discuss the driving mechanisms and wave properties of these EUV waves in more detail.

\subsection{Prominence Eruption, CME and the CME-Driven EUV wave}

The prominence showed a loop-like shape over the northwest limb; its eruption experienced three distinct phases: slow rising, fast rising, and violent eruption phases (see Figure 1 (b)-(d) and (g), and the animation available in the online journal). The slow rising phase was from 18:00 UT to 18:06 UT, and the prominence rose slowly at a speed of about $7 \mathrm{~km} \mathrm{~s}^{-1}$. The fast rising phase was from 18:06 UT to 18:16 UT, and the prominence rose quickly was about $64 \mathrm{~km} \mathrm{~s}^{-1}$.

\footnotetext{
${ }^{1}$ http://cdaw.gsfc.nasa.gov/CME_list
} 

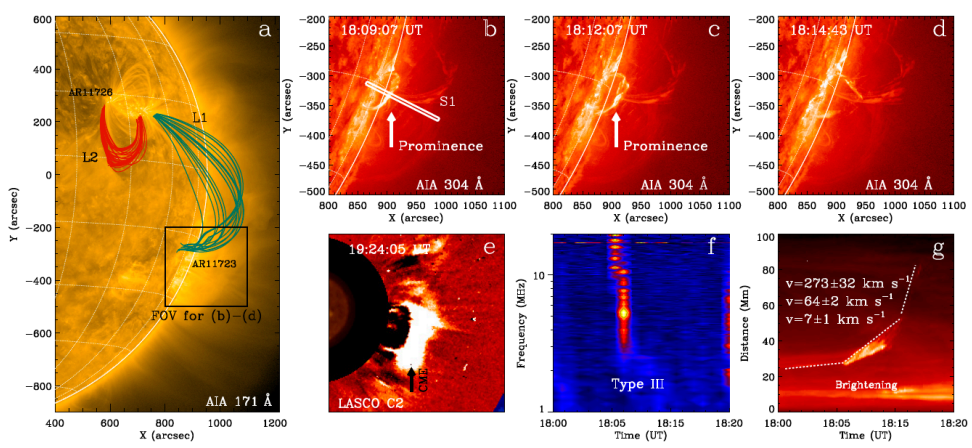

Figure 1. Coronal environment before the eruption in AIA $171 \AA$ (a) and the evolution of the eruption prominence (b)-(d), as well as the associated CME (e) and type III radio burst (g). Time-distance stack plot made from rectangle slice, labeled as "S1" in (b), extending from flare epicenter in AIA $304 \AA$ for eruption prominence (g). Note that the white arrows point to the eruption prominence, while the CME front is pointed out by the black arrow. Two groups of loop systems labeled as "L1" and "L2" in panel (a), are highlighted by extrapolated magnetic field lines.

After 18:16 UT, the prominence erupted violently at an average speed of about $273 \mathrm{~km} \mathrm{~s}^{-1}$. At 18:06 UT, the beginning of the fast rising phase of the prominence eruption, a type III radio burst was detected by the WAVES, and the prominence body became brighter than before (see Figure 1 (f) and (g)). Generally, type III radio bursts are considered to be a signature of accelerated energetic electrons streaming, channeled along open magnetic field lines, attributed to the reconfiguration of an unstable coronal magnetic field (Kozarev et al. (2011); Reid and Ratcliffe (2014); Gao et al. (2014); Li, Ning, and Zhang (2015); Shen et al. (2017b)). The type III radio burst observed here appeared at about $13 \mathrm{MHz}$, and it rapidly drifted to $3 \mathrm{MHz}$; this suggests the start of magnetic reconnection in the eruption and the propagation of accelerated energetic electrons from lower corona to higher altitude. In the wake of the ejected prominence, the flare reconnection is believed to occur between two legs of the envelope with a vertical current sheet inside, resulting in the further acceleration upward of prominence (Moore et al. (2001); Kliem and Török (2006); Karpen, Antiochos, and DeVore (2012)).

During the fast rising phase of the prominence, one can identify the CME bubble in the low corona over the southwest limb of the solar disk as displayed in the top row of Figure 2, which showed as a well-defined bright, sharp circular boundary. A large-scale EUV wave appeared ahead of the CME bubble a few minutes after the formation of the CME bubble (see Figure 2 (b)). In the meantime, diffuse bright wavefront can also be observed on the solar surface (see green arrows in Figure 2 (a) and (b)). The on-disk propagating EUV wave was further interacted with the remote active region AR11726 in the northern hemisphere, after which a reflected EUV wave appeared on the southeast side of AR11726 (see the blue arrows in Figure 2 (c)). Here, both the on-disk and radial propagating EUV waves should be driven by the CME, as those have been reported in previous studies (e.g., Veronig et al., 2010; Shen and Liu, 2012c; Shen et al., 2020). To analyze the kinematics of the EUV wave ahead of the CME bubble, we selected three azimuthal paths, labeled as "C1-C3" in Figure 2, to 

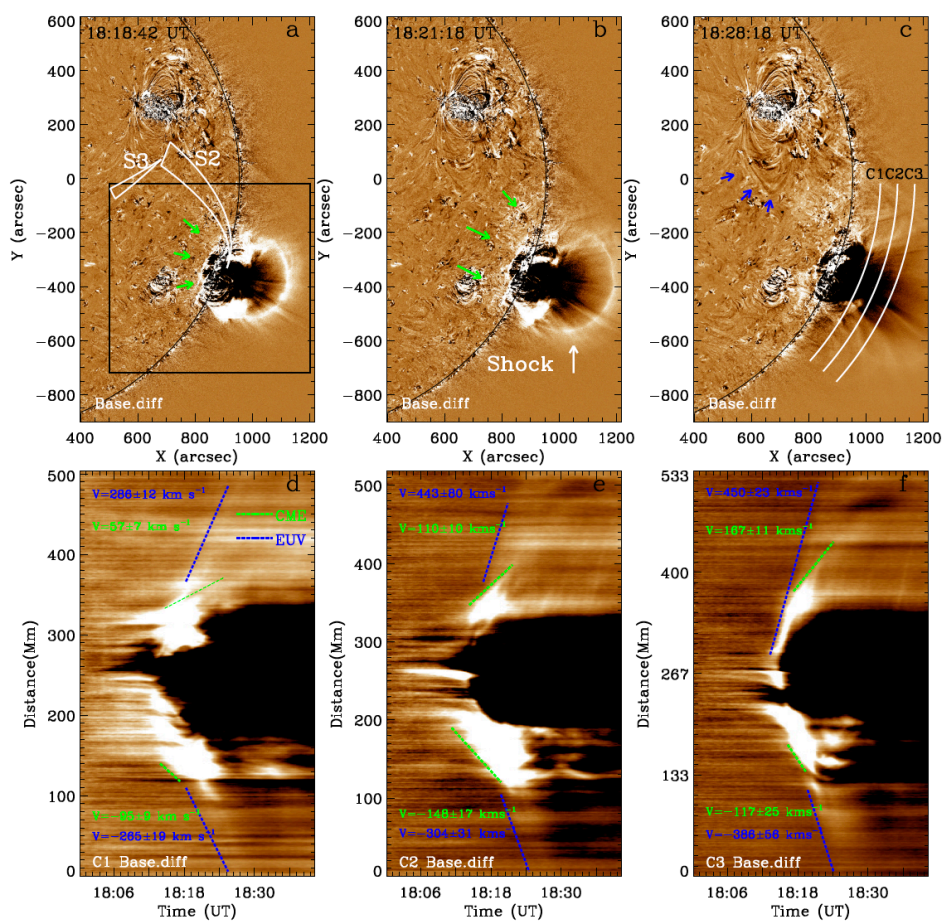

Figure 2. The evolution of the large-scale EUV wave in AIA $193 \AA$ base-difference images (top), and time-distance stack plots (bottom) for the three azimuthal paths labeled as "C1", "C2", and "C3" in panel (c). In panel (b), a shock ahead of the CME bubble is identified by white arrow. In panels (d)-(f), the moving features of the CME bubble and its lateral EUV wave are respectively outlined by the green and blue dashed lines, and their corresponding propagating velocities, determined by applying linear fits, are written with corresponding colors. The green arrows indicate the wavefront of the on-disk propagating EUV wave component, while the blue arrows mark the reflation wavefront. The black box denotes the FOV of panels (a)-(c) in Figure 4.

generate TDSPs from the AIA $193 \AA$ A base-difference images. Here, the azimuthal paths $\mathrm{C} 1-\mathrm{C} 3$ are part of concentric circles centered at the center of the solar disk, and their height above the disk limb are 60,100, and $140 \mathrm{Mm}$, respectively. The TDSPs generated along paths C1-C3 are displayed in Figure 2 panels (d)-(f). In each of TDSPs, the boundary of the CME bubble can be identified as a paired bright bidirectional oblique stripes, and they are indicated by the green dotted lines. Based on these TDSPs, the lateral expansion speeds in south and north directions are measured to be $95-148 \mathrm{~km} \mathrm{~s}^{-1}$ and $57-167 \mathrm{~km} \mathrm{~s}^{-1}$, respectively. The EUV wave ahead of the CME bubble can well be identified in these TDSPs, they are observed fainter than the CME bubble. The EUV wave firstly mixed with the CME bubble, it became clear until the expansion of the CME bubble started to slow down at about 18:18 UT. The generation of the EUV wave was directly separated from the outer edge of the CME bubble, and it propagated at a speed of about $265-450 \mathrm{~km} \mathrm{~s}^{-1}$, which is faster than the CME bubble about 2- 

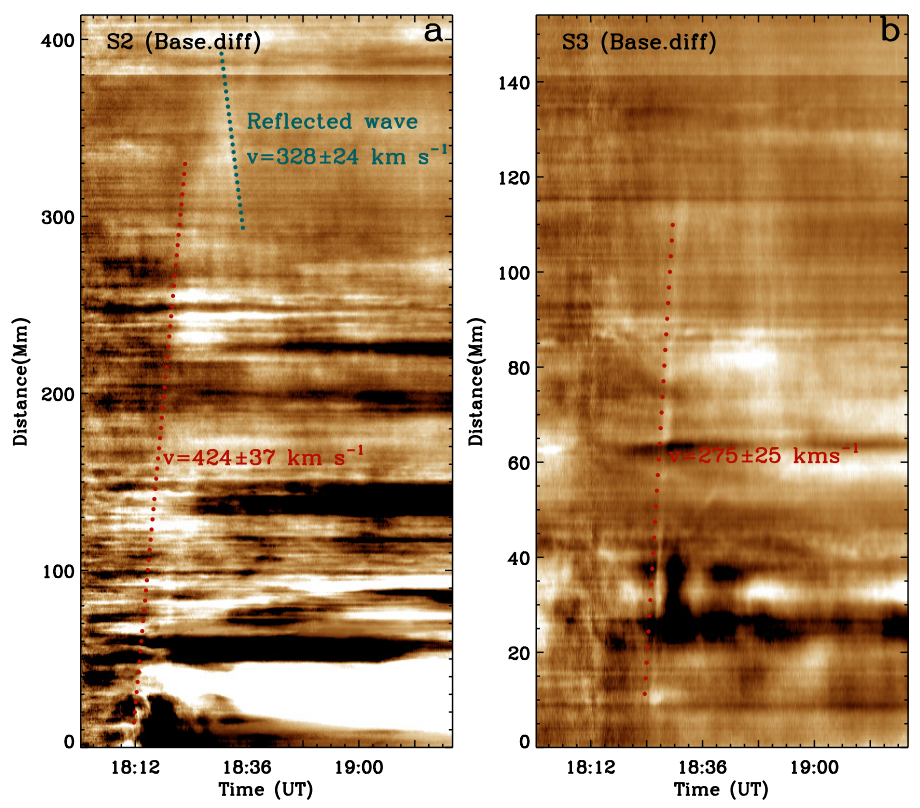

Figure 3. Time-distance stack plots for the two sectors, labeled as "S2" and "S3" in Figure 2, in AIA $193 \AA$ base-difference images. The velocities of the wavefronts are determined by using linear fits and they corresponding speeds are written around them.

4 times in the low corona. These observational results suggest that the observed EUV wave ahead of the CME bubble was driven by the expansion motion of the CME bubble, consistent with previous observations (e.g., Ma et al., 2011; Liu et al., 2012; Cheng et al., 2012; Liu, Zhu, and Zhao, 2019).

The on-disk propagating EUV wave component showed a semicircular shape and propagated oriented to the northeast direction. It interacted with AR11726 and produced a reflected wave propagated oriented to the southeast direction. To quantify the kinematics of these on-disk propagating EUV waves, we generated two TDSPs along sectors S2 and S3 as indicated in Figure 2 (a). We use AIA 193 $\AA$ base-difference images to generate the TDSPs, and the results are displayed in Figure 3. Note that the origin of sector S2 is located near the flare epicenter and orient to the loop system L2, while S3 is along the propagation direction of the reflected wave. In the TDSP along S2 (Figure 3 (a)), one can identify the start time of the on-disk propagating EUV wave was about 18:12 UT. The EUV wave showed obvious deceleration during the propagation, and the average projection speed is measured to be about $424 \mathrm{~km} \mathrm{~s}^{-1}$. At 18:24 UT, the EUV wave interacted with AR11726 and produced a reflected wave that propagated in the opposite direction of the primary EUV wave. Along S2, the reflected EUV wave had a speed of about $330 \mathrm{~km} \mathrm{~s}^{-1}$, significantly slower than the incident EUV wave. To more precisely measuring the speed of the reflected wave, we generate a new TDSP along S3 (Figure $3(\mathrm{~b})$ ). This path is perpendicular to 
the propagation direction of the reflected wave. It can be seen that the reflected wave also showed a deceleration, and had an average speed of about $275 \mathrm{~km} \mathrm{~s}^{-1}$.

\subsection{Flare-Ignited Quasi-Periodic EUV Wave Trains}

Besides the single pulse EUV waves ahead of the CME bubble and on the solar surface, it is striking that multiple successive arc-shaped wavefronts were observed within the CME bubble during the impulsive phase of the flare. We show this large-scale quasi-periodic wave train in Figure 4 with AIA $193 \AA$ running difference images (see the white arrows and the animation available in the online journal). The wavefronts emanated continuously from the flare epicenter and exhibited as a series of concentric semicircles resembling the shape of the CME bubble; they propagated outwardly and gradually disappeared when they reached up to the height of the boundary of the CME bubble. Using the AIA $193 \AA$ running difference images, we made a TDSP along the rectangle in Figure 4 (a) to study the kinematics of the large-scale quasi-periodic wave train inside the CME bubble, and the result is displayed in Figure 4 (d). In this TDSP, the brightest stripe is the boundary of the CME bubble, and the EUV wave ahead of the CME can be identified as a relatively fainter stripe on the left of the CME one. The large-scale quasi-periodic EUV wave train can be recognized as a series of weak stripes on the right of the CME one. By applying quadratic fit to these stripes, we obtain the average speed of the CME bubble and the large-scale quasi-periodic wave train were about $300 \mathrm{~km} \mathrm{~s}^{-1}$ and $1100 \mathrm{~km} \mathrm{~s}^{-1}$, respectively. The deceleration and period of the large-scale quasi-periodic wave are estimated to be $\alpha=-4.1 \mathrm{~km} \mathrm{~s}^{-2}$ and 120 seconds, respectively. Here, we note that the speed of the large-scale quasi-periodic wave train was significantly faster than the CME bubble and the single EUV wave ahead of the CME. In addition, the large-scale quasi-periodic wave train was behind of the CME bubble, and its appearance time was delayed about 4 minutes with respect to that of the rapidly rising phase of the CME bubble. Therefore, the large-scale quasi-periodic wave train was unlikely driven by the CME expansion as those have been documented in the literature (Warmuth, 2015; Shen et al., 2020).

Along the closed-loop system L1, we observed another quasi-periodic fast propagating EUV wave train that can well be identified in the AIA $171 \AA$ running difference images (see Figure 5 (a)-(c) and the animation available in the online journal). Compared with the large-scale quasi-periodic EUV wave train inside the CME bubble, this narrow quasi-periodic EUV wave train also emanated continuously from the flare epicenter, but it had a relatively smaller angular extent and its propagation was obviously guided by the coronal loop rather than confined within the CME bubble. These observational characteristics make us believe that the two observed quasi-periodic EUV wave trains were two independent EUV wave trains, but both of their generations were possibly associated with the flare. Here, the narrow quasi-periodic EUV wave train should be a so-called QFP magnetosonic wave as those reported in previous studies (e.g., Liu et al., 2011; Yuan et al., 2013; Shen and Liu, 2012b; Shen et al., 2013a; Miao et al., 2021). The propagations of such QFP waves are typically trapped in coronal loops acting as waveguides (high density, low Alfvén speed). 


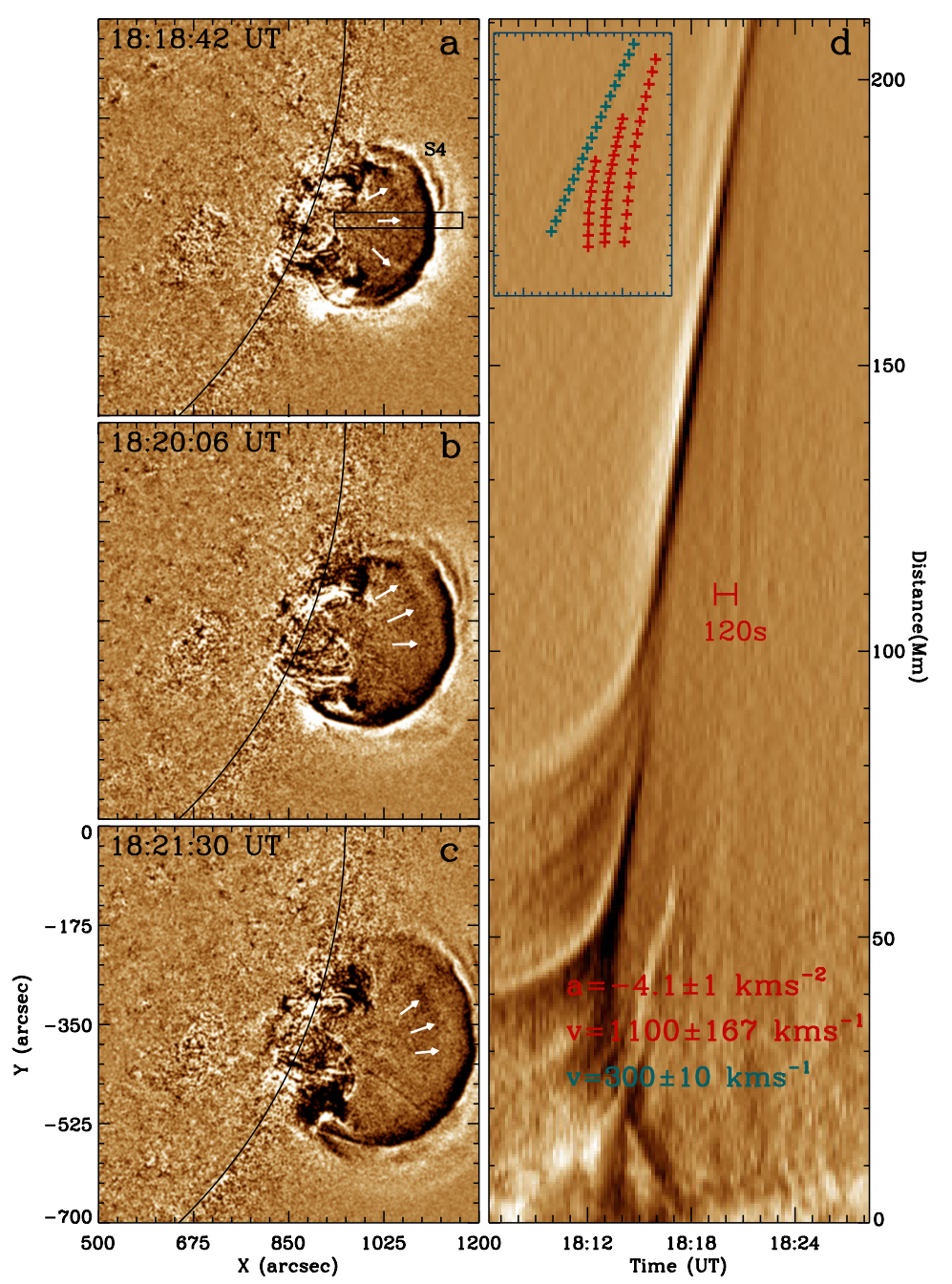

Figure 4. Evolution of the large-scale quasi-periodic EUV wave running inside the CME bubble. Panels (a)-(c) show the evolution of wavefront in AIA $193 \AA$ running-difference images, in which the white arrows point to the wavefronts. The FOV of panels (a)-(c) are the region indicated by the black box in Figure 2 (a). Panel (c) shows the AIA $193 \AA$ running-difference time-distance stack plot along a rectangle slice, labeled as "S4" in panel (a). The kinematic parameters of the CME bubble and the wavefronts of the large-scale quasi-periodic EUV wave are get from quadratic fits of the brighten ridges, labeled in panel (d). To show the wavefronts clearly, we outline their tracks into the top-left corner of panel (d).

Generally, QFP magnetosonic waves often appear during the impulsive phases of the associated flares. In the present case, the narrow quasi-periodic EUV wave train can be observed from about 18:24 UT to 19:12 UT, lasting up to 50 minutes from the flare impulsive phase to the decay phase. The TDSPs are generated by using the AIA $171 \AA$ and $193 \AA$ images along the S5 as indicated in Figure 5 (a), and the result are displayed in Figure 5 (d) and (e), respectively. In AIA $171 \AA$ TDSP, one can identify many bright parallel stripes that represent the 
propagating wavefronts of the QFP wave train. These wavefronts can also been found in AIA $193 \AA$ TDSP, but they are relatively fainter than it detected in AIA $171 \AA$ (see Figure 5 (d)(e) and the animation in the accompanying online material). By applying a linear fit to the stripes, we obtain the average speeds of the narrow quasi-periodic EUV wave train was about $475 \mathrm{~km} \mathrm{~s}^{-1}$.

The first strong stripe in Figure 5 (d) represents the EUV wave ahead of the CME bubble, which was trapped by the closed-loop system L1. It propagated along the entire loop system and produced a reflected wave during its interaction with the remote footpoint of the loop system. The EUV wave propagated at an average speed of about $450 \mathrm{~km} \mathrm{~s}^{-1}$; it was reflected at the remote footpoint of the loop at about 18:27 UT. The reflected wave propagated at a speed of about 320 $\mathrm{km} \mathrm{s}^{-1}$. It is noted that the generation of the reflected wave along the loop system delayed the on-disk reflected EUV wave by about 3 minutes, although its average speed was faster than that of the on-disk EUV wave. A alternative explanation could be that the EUV wave trapped in the loop propagated a longer distance, as the loop system should be a curving structure. Generally, EUV waves show broadening wavefronts and decreasing amplitudes during their propagation. For the present case, the intensity amplitudes and propagation speeds of the EUV wave at different sections of the loop showed an abnormal varying pattern, i.e., the amplitude and speed were firstly decreasing continuously from the origin to the middle section of the loop, then they followed an increasing trend from the middle section to the remote end of the loop system (see Figure 5 (d)). These features are typical characteristics of large-scale fast-mode magnetosonic waves steepened to form freely propagating shocks traveling along loops (Mann, 1995). The intensity amplitude of an EUV wave is determined by the energy and geometric of the waveguide, and the propagating speed is related to the amplitude (Warmuth, 2010, 2015). Obviously, the middle section of the loop system had a broader cross-section than that near the two footpoints. As the EUV wave propagated from one end of the loop to the middle section, the wave energy spread to a broader extent and therefore led to a decrease of the intensity amplitude (Yuan et al., 2013). During this period, the EUV wave evolved from a shock or non-linear fast-mode magnetosonic wave into a linear fast-mode magnetosonic wave. From the middle section of the loop to the other end, the wave energy and amplitude experience a reverse process, and the linear fast-mode magnetosonic wave became a non-linear fast-mode magnetosonic wave or shock close to the remote end of the loop. The speed of a fast-mode magnetosonic wave is directly proportional to the magnetic field strength of the medium in which it propagates. For the closed-loop system L1, the magnetic field strengths close to the two ends should be greater than that close to the apex. Therefore, the wave speed was naturally experienced first decreasing and then increasing processes. In practice, the speed of the fast-mode magnetosonic wave is also determined by plasma density and sound speed, as well as the projection effect can also be important. Therefore, the actual measured speed of the EUV wave was inflected for many reasons, such as the inhomogeneous distribution of the plasma density and magnetic field strength, local sound speed, and projection effect. 

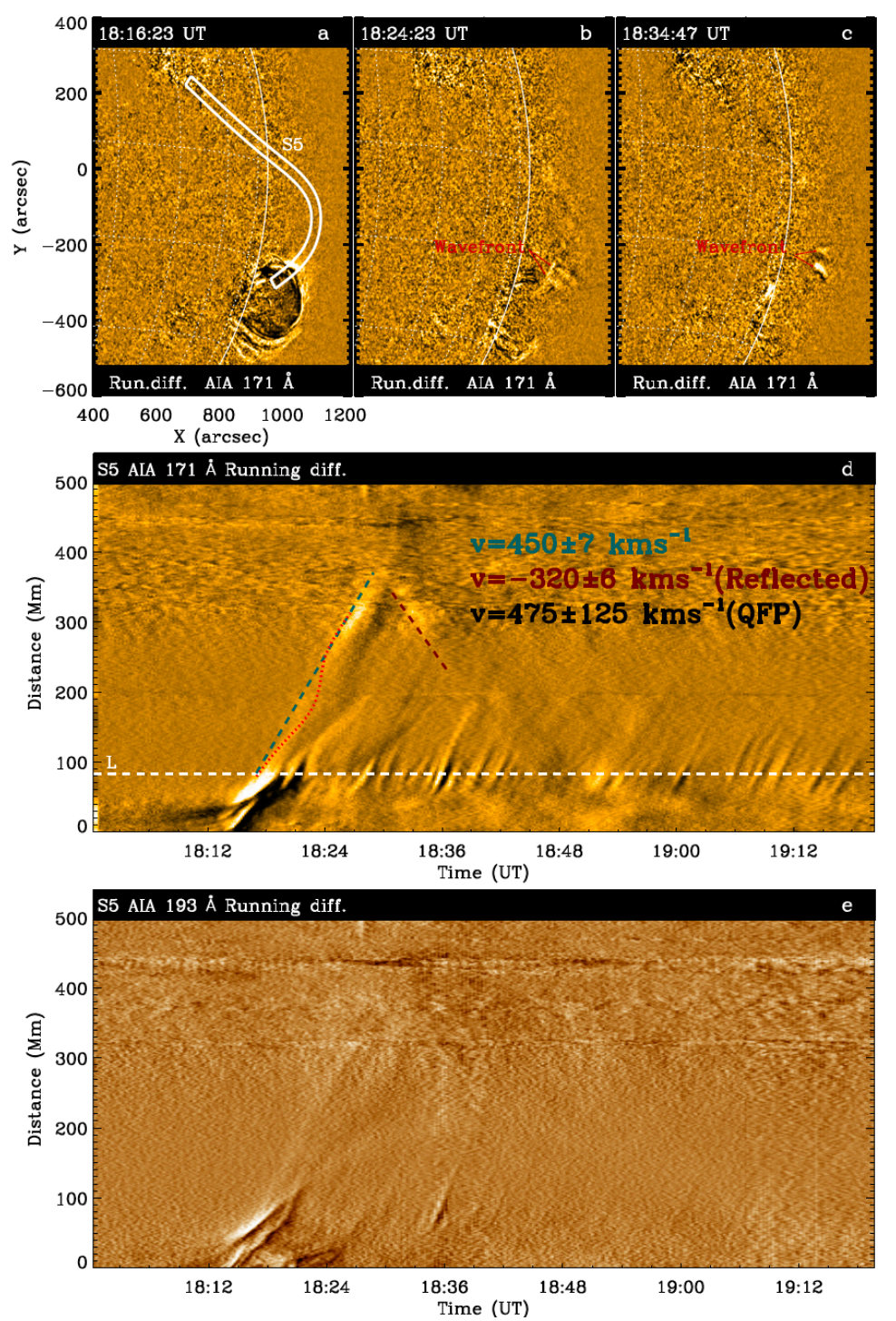

Figure 5. The evolution of the narrow quasi-periodic EUV waves running along L1 in AIA $171 \AA$ A running-difference images (a)-(c). The red arrows point to the wavefronts. Panels (d) and (e) respectively show the time-distance stack plots constructed from AIA $171 \AA$ and 193 A running-difference images along the white slice labeled as "S5" in panel (a), in which the horizontal white dashed line indicates the position whose intensity profile is used to analyze the periodicity of the narrow quasi-periodic wave trains, while the red and blue dashed lines denote the linear fits to the wave ridges. The red dotted curve line roughly indicates the trend of ridge. The average speeds of the incident and reflected EUV wave are $450 \pm 7 \mathrm{~km} \mathrm{~s}^{-1}$ and $320 \pm 6 \mathrm{~km} \mathrm{~s}^{-1}$, respectively. The average velocity of the narrow quasi-periodic EUV waves is $475 \pm 125 \mathrm{~km} \mathrm{~s}^{-1}$ 


\subsection{Periodicity of the Quasi-Periodic EUV Wave Trains and the Flare}

The periodicity of the quasi-periodic EUV wave trains and the associated flare is analyzed in detail by using the wavelet analysis method (Torrence and Compo, 1998). Figure 6 (a) displays the GOES soft X-ray flux profile in the energy band of $0.5-4 \AA$. Since this flare lacks hard X-ray observations and limb occultation of the flare ribbons in the UV and EUV observations, we use the time derivative of the GOES 0.5-4 $\AA$ soft X-ray flux to analyze the periodicity of the flare. According to the Neupert effect (Neupert, 1968), such time derivative soft X-ray fluxes can be viewed as a proxy of the corresponding hard X-ray fluxes that reflect the variation of non-thermal particles accelerated by the magnetic reconnection

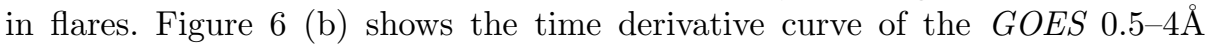
soft X-ray flux during the flare. We subtracted a 400 seconds smoothed curve (blue) to detrend the signal and the result is overlaid in Figure 6 (c). Using this signal as input, we generated the wavelet spectrum, and the result is displayed in Figure 6 (c). One can identify several main periods in the wavelet spectrum, they appeared mainly during two time spans of 18:10-18:40 UT and 18:50-19:15 UT. It is determined based on the global power of the wavelet spectrum map that the main dominated periods in the flare were about 85 and 125 seconds, which is similar to the result analyzed the hard X-ray (Li et al., 2017).

Since the wave signal of the large-scale quasi-periodic EUV wave train inside the CME bubble was too weak, we failed to obtain its period with the wavelet technique. As indicated by the short red bar in Figure 4 (c), we directly estimated its period to be about 120 seconds by measuring the time span during two neighboring wave crests. The wave signal of the narrow quasi-periodic EUV wave train was relatively stronger. Therefore, we analyzed its periodicity by using the intensity profile varying with time, which can be obtained by extract the intensity profiles along the horizontal lines marked "L" as shown in Figure 5 (d). We also use the detrended intensity profile as the input for the wavelet procedure; it is also overlaid in the wavelet spectrum map as a yellow curve in Figure 6 (d). In the same way, we determined the periods of the EUV wave train based on the peak of the global power curve of the wavelet spectrum. It is obtained that the main period of the narrow quasi-periodic EUV wave was about 113 seconds, which is roughly similar to the result reported by Kumar, Nakariakov, and Cho (2017). Obviously, all the obtained periods of the largescale and narrow quasi-periodic EUV wave trains were the same with those of the pulsations in the flare, suggesting their common origin.

\subsection{Wave energy and seismology application}

The energy flux $\mathcal{F}$ per unit area and time carried by the waves can be estimated from the kinetic energy of the perturbed plasma using the expression $\mathcal{F}=\frac{1}{2} \rho(\delta v)^{2} v_{p h}$, where $\rho$ is the mass density, $v$ is the disturbance speed of local plasma, and $v_{p h}$ is the phase speed of the EUV wave (Aschwanden, 2004a). It is generally believed that the observed EUV emission intensity is proportional to the square of the plasma density, i.e., $I \propto \rho^{2}$. Thus the density modulation 

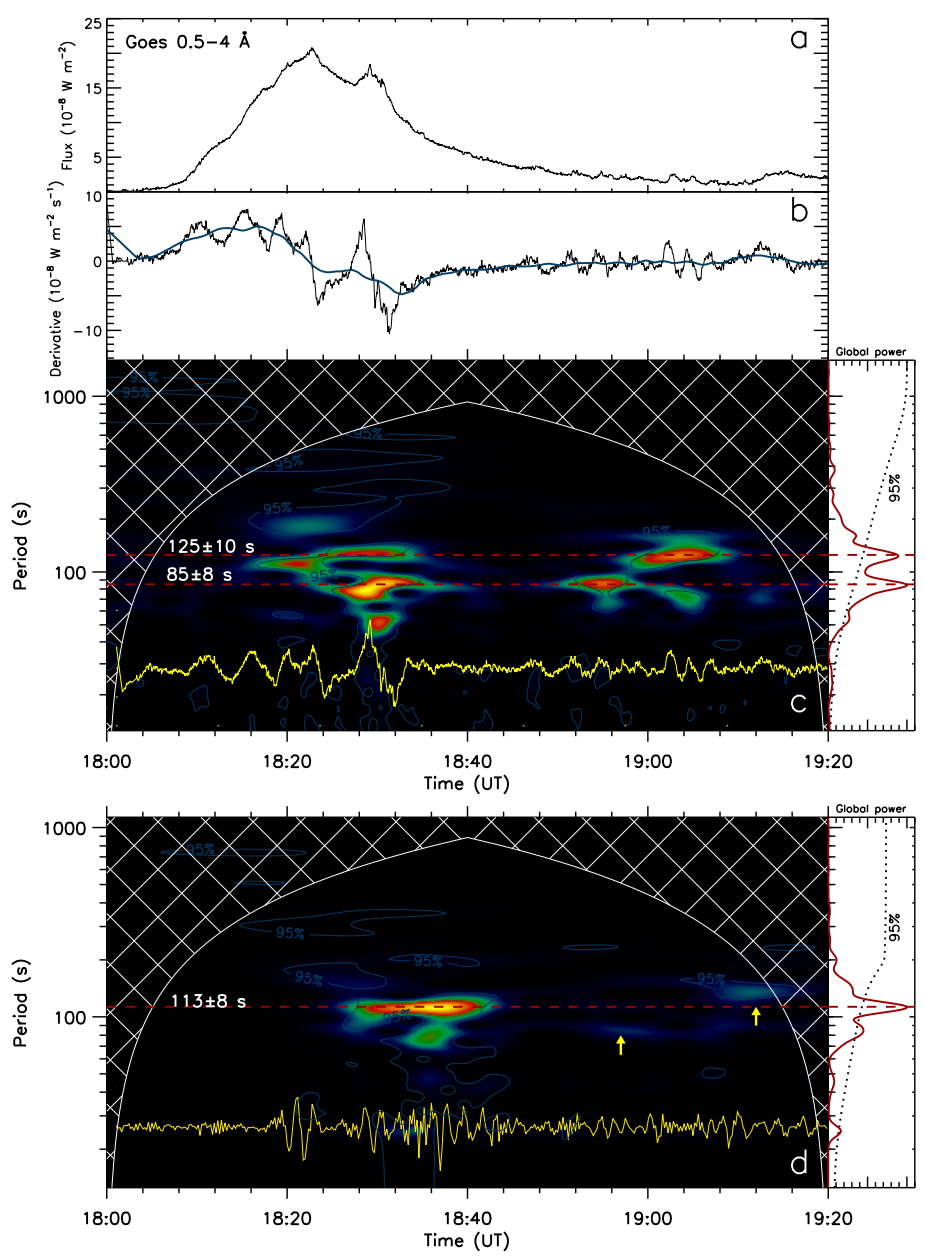

Figure 6. Periodicity analysis for the narrow quasi-periodic EUV wave and the associated flare pulsation. Panels (a)-(b) are GOES 0.5-4 $\AA$ soft X-ray flux and its time derivative, respectively. The yellow curve in panel (c) is smooth/detrended time derivative signal of the GOES 0.5-4 A soft X-ray flux after subtracting the blue smooth curve, while the yellow curve in panel (d) is the smooth/detrended intensity profile of the horizontal white dashed line labeled as "L" in Figure 5 (d), and their corresponding wavelet power spectrums are displayed in panel (c) and (d), respectively. The dotted line in global power indicates the $95 \%$ significance level, while the red horizontal dashed lines mark the periods. 
Table 1. Physical Parameters of the Various EUV Waves

\begin{tabular}{lccccc}
\hline Various EUV Waves & $v\left[\mathrm{~km} \mathrm{~s}^{-1}\right]$ & $I / I_{\mathrm{O}}$ & $\delta I$ & $M$ & $\mathcal{F}\left[\mathrm{erg} \mathrm{cm}^{-2} \mathrm{~s}^{-1}\right]$ \\
\hline EUV wave on the solar surface & 424 & 1.14 & $14 \%$ & 1.05 & $0.6 \times 10^{5}$ \\
On-disk reflected wave & 275 & 1.08 & $8 \%$ & 1.03 & $0.05 \times 10^{5}$ \\
EUV wave along the closed-loop & 450 & 1.17 & $17 \%$ & 1.04 & $4.18 \times 10^{5}$ \\
Reflected wave along the closed-loop & 320 & 1.15 & $15 \%$ & 1.03 & $1.2 \times 10^{5}$ \\
\hline Large-scale quasi-periodic EUV wave train & 1100 & 1.15 & $15 \%$ & 1.05 & $12.0 \times 10^{5}$ \\
Narrow quasi-periodic EUV wave train & 475 & 1.05 & $5 \%$ & 1.01 & $0.43 \times 10^{5}$ \\
\hline
\end{tabular}

$\delta \rho$ and the observed emission intensity variation $\delta I\left(\delta I=\left(I-I_{0}\right) / I_{0}\right)$ have the relation $\delta \rho / \rho=\delta I /(2 I)$, where $I$ and $I_{0}$ respectively are highest amplitude and background intensity. Following Liu et al. (2011) and assuming that the observed intensity variation $\delta I$ stems from the density modulation $\delta \rho$ and using $\delta v / v_{p h} \geqslant \delta I /(2 I)$, then the energy flux $\mathcal{F}$ of the perturbed plasma could be rewritten as $\mathcal{F} \geqslant \frac{1}{8} \rho(\delta I / I)^{2} v_{p h}^{3}$. Here we assume that the electron-number density of the corona and the waveguiding loops are $n_{e}=1.5 \times 10^{8} \mathrm{~cm}^{-3}$ (Aschwanden, 2004a) and $n_{e}=6 \times 10^{8} \mathrm{~cm}^{-3}$ (Aschwanden, 2004b), respectively. Meanwhile, we take the average phase speeds for the observed large-scale EUV wave, the large-scale quasi-periodic EUV wave train, and the narrow quasiperiodic EUV wave train are $424 \mathrm{~km} \mathrm{~s}^{-1}, 1100 \mathrm{~km} \mathrm{~s}^{-1}$ and $475 \mathrm{~km} \mathrm{~s}^{-1}$, and their corresponding relative intensity perturbations of $\delta I$ are $14 \%, 15 \%$ and $5 \%$, respectively. Then we arrive at the energy fluxes $\mathcal{F}$ for the three types of EUV waves are $0.6 \times 10^{5} \mathrm{erg} \mathrm{cm}^{-2} \mathrm{~s}^{-1}, 12.0 \times 10^{5} \mathrm{erg} \mathrm{cm}^{-2} \mathrm{~s}^{-1}$ and $0.43 \times 10^{5} \mathrm{erg} \mathrm{cm}^{-2}$ $\mathrm{s}^{-1}$, respectively. With the same method, we calculate the energy flux of the other waves. The corresponding parameters used in our calculation and the obtained results are all listed in table 1 . We find that the energy flux density sorted in decrease turn as followed: Large-scale quasi-periodic EUV wave train, EUV wave along the closed-loop, reflected wave along the closed-loop, EUV wave on the solar surface, narrow quasi-periodic EUV wave train, on-disk reflected wave. At the meantime, the energy density of reflected waves are significant smaller than their corresponding incident waves. This is probably due to the loss in energy during the interaction, or only a part of the incident waves were reflected. It is noted that the energy flux density of the trapped EUV wave along the closedloop is about seven times of that on the solar surface. Because the two EUV waves were all excited by the expansion of the CME bubble, they should have a similar energy flux density during their initial stage. The large difference between the two EUV waves may suggest that the energy loss rate of the EUV wave along the closed-loop was slower that that on the solar surface, i.e., the loop waveguide can effectively maintain the wave energy during the propagation. In addition, the energy flux density of the large-scale quasi-periodic EUV wave train inside the CME bubble is thirty times of the narrow quasi-periodic EUV wave along the closed-loop system, indicating their distinctively different properties of the two types of EUV wave trains.

In the low $\beta$ (the ratio of plasma pressure to magnetic pressure) corona, one can estimate the Mach number of fast-mode magnetosonic waves based on the measurable emission density jump parameter by assuming $I \propto \rho^{2}$, i.e., 
Table 2. Event Timeline

$\begin{array}{ll}\text { 18:00 UT } & \text { Slow rising of the prominence } \\ \text { 18:06 UT } & \text { Fast rising of the prominence } \\ \text { 18:12 UT } & \text { Start of the EUV wave ahead of the CME bubble } \\ \text { 18:16 UT } & \text { Appearance of the large-scale quasi-periodic EUV wave train inside the CME bubble } \\ \text { 18:20 UT } & \text { Appearance of the narrow quasi-periodic EUV wave train along the closed-loop } \\ \text { 18:24 UT } & \text { Impingement of the on-disk EUV wave upon the remote active } \\ & \text { Appearance of the reflected EUV waves on the surface and along the closed-loop }\end{array}$

$X=\frac{\rho}{\rho_{0}}=\sqrt{\frac{I}{I_{0}}}$ (Priest, 1982). The expression for the Mach number calculation for a fast-mode magnetosonic wave propagating perpendicular (along) coronal loops can be written as $M=\sqrt{\frac{X(X+5)}{(2(4-X))}}\left(M=X^{\frac{1}{2}}\right)$. Based on the measured emission density jump values of the EUV waves observed in the present event, we obtained their Mach numbers (see Table 1). It can be seen that the Mach numbers of various EUV waves are in the range of 1.01-1.05, indicating that these waves are all weakly shocked fast-mode non-linear magnetosonic waves. The Mach number for the EUV wave along the solar surface is about 1.05, which for the EUV wave trapped in the closed-loop is about 1.04. It is noted that the Mach numbers of the reflected EUV waves on the solar surface and along the closed-loop are relatively smaller than their corresponding incident waves. For the quasi-periodic EUV wave trains, the Mach number of the large-scale quasiperiodic EUV wave train inside the CME bubble is significantly larger than that of the narrow quasi-periodic EUV wave train along the closed-loop.

With the observational phase speed of the propagating waves described above, we can estimate the local magnetic field strengths of the close-loop and quiet-Sun corona where the EUV waves propagated. The sound speed $c_{s}$ can be calculated from the formula $c_{s}=147 \sqrt{\frac{\mathrm{T}_{e}}{1 \mathrm{MK}}}$ (Aschwanden, 2004b) in a fully ionized plasma. For the formation temperature $\left(T_{e}\right)$ of the AIA $193 \AA$ channel, which is at 1.6 MK. Therefore, we can obtain the corresponding sound speed $\left(c_{s}\right)$ is about $185 \mathrm{~km} \mathrm{~s}^{-1}$. Based on the formula $v=\sqrt{c_{s}{ }^{2}+v_{A}{ }^{2}}$ and the measured speed of the EUV wave $\left(424 \mathrm{~km} \mathrm{~s}^{-1}\right)$, we can estimate that the Alfvén speed and the magnetic strength in the quiet-Sun corona are respectively about $382 \mathrm{~km} \mathrm{~s}^{-1}$ and 2.3 Gauss, by assuming that the wave-vector of the on-disk propagating EUV wave was perpendicular to the magnetic field. Here, $v_{A}$ is the local Alfvén speed, which is written as $v_{A}=\frac{B}{\sqrt{4 \pi \rho}}=\frac{B}{\sqrt{4 \pi \mu m_{p} 1.92 n_{e}}}$ (Priest, 1982).

We can estimate the magnetic field strength of the closed-loop system L1 based on the trapped EUV wave and the narrow quasi-periodic EUV wave train, respectively. For a fast-mode magnetosonic wave propagating along the magnetic field, its speed should be the same with the local Alfvén speed. So, we can obtain the magnetic field strength of the coronal loop based on the formula $v_{A}=\frac{B}{\sqrt{4 \pi \rho}}$. However, as that had been pointed out in Shen et al. (2019), the

${ }^{2} \mu=1.27$ is the mean molecular weight in the corona, $m_{p}=1.67 \times 10^{-24} \mathrm{~g}$ is the proton mass. 
result directly obtained by using the measured speed of EUV wave should be larger than the true value, since the observed EUV waves should be shocks or non-linear fast-mode magnetosonic waves. To derive the real magnetic field of the coronal loop, one should first obtain the linear fast-mode magnetic speeds by dividing the measured speeds of the EUV waves by their Mach numbers. The Mach number of an EUV wave can be calculated by utilizing the formula $M=\sqrt{\frac{n}{n_{0}}}$ (Priest, 1982), where $n$ and $n_{0}$ are the peak and background densities. We firstly measured the highest emission intensity amplitude $\left(\frac{I}{I_{0}}\right)$ of the EUV wave (1.17) and the narrow quasi-periodic EUV wave train (1.05) along the closed-loop system L1, then we can obtain the Mach numbers of the two EUV waves are respectively about 1.04 and 1.01 by assuming $I \propto \rho^{2}$ and the wavevectors of the EUV waves were parallel to the magnetic field. The average speed of the EUV wave (narrow quasi-periodic wave train) was 450 (475) $\mathrm{km} \mathrm{s}^{-1}$, based on which we finally obtain the magnetic field strength of the closed-loop system is about 5.2 (5.6) Gauss, consistent with the result estimated by Shen et al. (2019).

\section{Conclusion and Discussion}

Using high spatiotemporal resolution observations taken by the $S D O$, we studied three types of EUV waves launched in a single solar eruption accompanied by a GOES C3.0 flare, a prominence eruption and a CME. The waves include largescale single pulse EUV waves propagated simultaneously ahead of the CME bubble and on the solar surface, a large-scale quasi-periodic EUV wave train inside the CME bubble, and a narrow quasi-periodic EUV wave train along a transequatorial closed-loop system. Based on our analysis results, we propose that all these waves are fast-mode magnetosonic waves, in which the largescale single pulse EUV waves propagated ahead of the CME bubble and on the solar surface were excited by the expansion of the CME, while the largescale quasi-periodic EUV wave train inside the CME bubble and the narrow quasi-periodic EUV wave train along the closed-loop were probably launched by the intermittent energy releasing process in the flare. To make clear the time relationship among various eruption features of the event, we list the start times of each eruption step in Table 2.

Large-scale single pulse EUV waves associated with on-disk solar eruptions are generally mixed with the associated CME bubbles, thus it is difficult to distinguish their origin. Therefore, direct observational evidence for determining the relationship between EUV waves and CMEs is limited (Shen et al., 2017a). Alternatively, limb events are more suitable for such kind of study (Ma et al., 2011; Cheng et al., 2012). The present limb event provides a rare opportunity for us to diagnose the relationship between the EUV wave and the CME. It is demonstrated that the EUV wave ahead of the CME bubble was directly separated from the CME bubble during its fast expansion stage, and the formed EUV wave was faster than the CME 2-4 times at different heights. The observation also indicates that the formation height of the EUV wave was about 100 
Mm above the solar surface, consistent with previous stereoscopic observations (Delannée et al., 2014). Taken these observational results together, we propose that the rapid CME expansion compresses the surrounding plasma, resulting in the formation of the observed EUV wave (Liu et al., 2012; Cheng et al., 2012). Note that we did not detect the slower "wave" trailing behind of the fast EUV wave as predicted in Chen et al. (2002). Based on the speed relationship between the EUV wave and the associated CME, we tend to think that the CME bubble may correspond to the so-called slower pseudo wave component. EUV waves often interact with remote strong magnetic structures such as active regions or coronal holes, and wave effects such as reflection, refraction, and transmission can be expected at their boundaries (Gopalswamy et al., 2009; Olmedo et al., 2012; Kumar and Innes, 2013, 2015; Shen and Liu, 2012a,c; Shen et al., 2013b, 2019). The present event also showed a reflected EUV wave when the on-disk propagating EUV wave interacted with the active region AR11726. Its lifetime and traveling distance before disappear were about 6 minutes and $120 \mathrm{Mm}$, respectively. Besides, the large-scale EUV wave ahead of the CME bubble was partially trapped by the closed-loop system L1, it also showed reflected effect at the remote footpoint. The speeds of the reflected waves in the loop and on the solar surface were all decreased significantly with respect to their corresponding incident waves; this is possibly due to the loss of energy during the interaction. For the trapped EUV wave, it showed a changing intensity amplitude and speeds during its propagation along the loop, which reflects the variation of the physical parameters of the loop system. According to the conservation law of wave energy, we propose that the deceleration (acceleration) of the EUV wave is the result of energy disperse (concentrate) into a broad (narrow) cross-section waveguide, which leads to the increase (decrease) of the intensity amplitude.

The large-scale quasi-periodic EUV wave train observed inside the CME bubble had a projection speed of about $1100 \mathrm{~km} \mathrm{~s}^{-1}$, a period of about 120 seconds, and an intensity amplitude of about $15 \%$ higher than the background. The arcshaped wavefronts of the wave train were observed to be generated continuously from the flare epicenter and propagated upwardly in the radial direction; they became prominent in a higher altitude at a height greater than $50 \mathrm{Mm}$ from the solar surface, indicating their increasing amplitude since their origin. This seems to imply that we just observed the initial steeping stage of a typical fast-mode magnetosonic wave or shock (Warmuth, 2007). Since the wavefronts were inside the CME bubble and propagated radially as the CME bubble, they continuously disappeared when they reached up to the height of the frontal of the CME. Since the speed, amplitude, and intensity profile of the wave train are similar to those of typical large-scale EUV waves, we suggest that it should be a fast-mode magnetosonic wave.

How did the large-scale quasi-periodic EUV wave train originate? In the case of the piston-driven shock, the wavefront should be initially located ahead of the driver and then decouples from the driver and propagates freely in the supporting medium. In most studied cases, CMEs are proposed as the typical driver of large-scale single pulse EUV waves (Liu and Ofman, 2014; Warmuth, 2015; Shen et al., 2020), such as the one ahead of the CME bubble in the present event. For the large-scale quasi-periodic EUV wave train, not only appeared inside 
the CME bubble but also delayed the rapid expansion of the CME bubble by about 4 minutes. In addition, it was composed of multiple coherent wavefronts rather than a single pulse. These observational characteristics suggest that the observed large-scale quasi-periodic EUV wave train was unlikely excited by the associated CME. Since the observed EUV wave train has a similar period to the quasi-periodic pulsation of the associated flare, we more tend to regard that it was excited by the quasi-periodic pressure pulses launched by the intermittent energy releasing-process in the flare (Vršnak and Cliver, 2008). If in that case, to the best of our knowledge, the present event should be a rare case showing strong evidence for supporting the flare-ignited EUV wave scenario. A possible detection of flare-ignited EUV wave event was presented by Magdalenić et al. (2008) using radio observations, where the authors observed a type II radio burst which was well-timed with the associated flare but lagged the impulsive acceleration phase of the associated CME of about 10-20 minutes. Other ambiguous evidence were also documented as flare-ignited shocks, such as type II radio burst without any associated CME (Magdalenić et al., 2012), pseudo CMEs (Eselevich, Eselevich, and Zimovets, 2019), and null-point reconnection in fan-spine magnetic systems (Kumar, Innes, and Cho, 2016). Recent high spatiotemporal observations indicated that the generation of large-scale EUV waves does not need the presence of CMEs, they can be launched by coronal jets (Shen et al. (2018d)), sudden loop expansions due to the impingement of other eruptions (Shen et al., 2017a, $2018 \mathrm{c}, \mathrm{b})$, or loop expansion of the reconnected open loop in coronal jets ( $\mathrm{Su}$ et al., 2015).

We note that large-scale quasi-periodic EUV wave trains propagating across a large fraction of the solar surface have only been reported in two studies (Liu et al., 2012; Shen et al., 2019). Liu et al. (2012) first observed an ambiguous example in the 2010 September 8-9 event and they proposed that the multiple wavefronts were within a broad global EUV wave propagating ahead of the associated $\mathrm{CME}$, and they were driven by the strong lateral, downward expansion effect of the CME. For their scenario, it is hard to understand how can a single CME excite multiple large-scale EUV wavefronts. In addition, it is also difficult to understand the relationship between the multiple wavefronts and the broad global EUV wave. Shen et al. (2019) reported an unambiguous large-scale quasiperiodic EUV wave train on 2012 April 24, in which multiple coherent EUV wavefronts appeared consecutively at the periphery region of the active region and propagated simultaneously along a group of closed-loop system and on the solar surface. The authors found that the period of the wave train showed a large difference with the pulsations in the associated flare, but it was similar to the unwinding period of the helical thread of an erupting filament in the source region. Therefore, they proposed that the observed large-scale quasi-periodic EUV wave train was excited by the successive opening of the twisted threads of the eruption filament. Besides, the authors also proposed that such kind of EUV wave train should be different than the so-called QFP wave train due to several distinct observational characteristics that the latter do not have, including 1) the wave signal can be simultaneously observed at all the EUV channels of AIA, 2) the wavefronts propagate perpendicular to and parallel to coronal magnetic field lines, and 3) the intensity profiles and amplitudes of the wavefronts are more 
similar to typical large-scale EUV waves. Therefore, they called such kind of EUV wave as a large-scale quasi-periodic EUV wave train to show the difference between QFP wave trains.

The narrow quasi-periodic EUV wave train along the closed-loop system should be a typical flare-ignited QFP wave train as those have been documented in the literature (see, Liu and Ofman, 2014, , and references therein). Comparing with large-scale quasi-periodic EUV wave trains, the narrow quasi-periodic EUV wave train was only observed at the AIA $171 \AA$ channel with a small intensity amplitude of about 5\%, and propagated along the coronal loop. There is a main period in the narrow EUV wave train, which is of $113 \pm 8 \mathrm{~km} \mathrm{~s}^{-1}$. Obviously, the period of the narrow wave train was similar to the pulsation in the associate flare. This suggests that the narrow EUV wave train was possibly excited by the quasi-periodic pressure pulses launched by the intermittent energy-releasing process in the flare. It is noted that the appearance of the narrow quasi-periodic EUV wave train was slightly after the period signal detected in the flare, which indicates that some necessary time is needed for the wave signal to steep to a certain extent to be detected by $S D O /$ AIA. A similar wave signal was also detected after the flare duration (see Figure 6 (c) and the arrows in Figure 6 (d)). It is unclear this was caused by another small flare or small energy release process in the late phase of the main flare.

Acknowledgments We thank the anonymous referee for providing detailed suggestions that helped improve the paper. We also thank the SDO and SOHO teams for the data supply. This work is supported by the Yunnan Science Foundation (2017FB006), the National Key R\&D Program of China (2019YFA0405000), the Natural Science Foundation of China $(12173083,11922307,11773068,11633008)$, the Yunnan Science Foundation for Distinguished Young Scholars (202101AV070004), the Specialized Research Fund for State Key Laboratories, the Open Research Program of CAS Key Laboratory of Solar Activity (KLSA202017) and the West Light Foundation of Chinese Academy of Sciences.

\section{References}

Aschwanden, M.J.: 2004a, Physics of the Solar Corona. An Introduction. ADS.

Aschwanden, M.J.: 2004b, The Role of Observed MHD Oscillations and Waves for Coronal Heating. In: Walsh, R.W., Ireland, J., Danesy, D., Fleck, B. (eds.) SOHO 15 Coronal Heating, ESA Special Publication 575, 97. ADS.

Aschwanden, M.J., Fletcher, L., Schrijver, C.J., Alexander, D.: 1999, Coronal Loop Oscillations Observed with the Transition Region and Coronal Explorer. Astrophys. J. 520, 880. DOI. ADS.

Bougeret, J.-L., Kaiser, M.L., Kellogg, P.J., Manning, R., Goetz, K., Monson, S.J., Monge, N., Friel, L., Meetre, C.A., Perche, C., Sitruk, L., Hoang, S.: 1995, Waves: The Radio and Plasma Wave Investigation on the Wind Spacecraft. Space Sci. Rev. 71, 231. DOI. ADS.

Brueckner, G.E., Howard, R.A., Koomen, M.J., Korendyke, C.M., Michels, D.J., Moses, J.D., Socker, D.G., Dere, K.P., Lamy, P.L., Llebaria, A., Bout, M.V., Schwenn, R., Simnett, G.M., Bedford, D.K., Eyles, C.J.: 1995, The Large Angle Spectroscopic Coronagraph (LASCO). Solar Phys. 162, 357. DOI. ADS.

Chandra, R., Chen, P.F., Joshi, R., Joshi, B., Schmieder, B.: 2018, Observations of Two Successive EUV Waves and Their Mode Conversion. Astrophys. J. 863, 101. DOI. ADS.

Chen, P.F.: 2006, The Relation between EIT Waves and Solar Flares. Astrophys. J. Lett. 641, L153. DOI. ADS. 
Chen, P.F., Wu, Y.: 2011, First Evidence of Coexisting EIT Wave and Coronal Moreton Wave from SDO/AIA Observations. Astrophys. J. Lett. 732, L20. DOI. ADS.

Chen, P.F., Fang, C., Shibata, K.: 2005, A Full View of EIT Waves. Astrophys. J. 622, 1202. DOI. ADS.

Chen, P.F., Wu, S.T., Shibata, K., Fang, C.: 2002, Evidence of EIT and Moreton Waves in Numerical Simulations. Astrophys. J. Lett. 572, L99. DOI. ADS.

Cheng, X., Zhang, J., Olmedo, O., Vourlidas, A., Ding, M.D., Liu, Y.: 2012, Investigation of the Formation and Separation of an Extreme-ultraviolet Wave from the Expansion of a Coronal Mass Ejection. Astrophys. J. Lett. 745, L5. DOI. ADS.

Cliver, E.W., Laurenza, M., Storini, M., Thompson, B.J.: 2005, On the Origins of Solar EIT Waves. Astrophys. J. 631, 604. DOI. ADS.

Delaboudinière, J.-P., Artzner, G.E., Brunaud, J., Gabriel, A.H., Hochedez, J.F., Millier, F., Song, X.Y., Au, B., Dere, K.P., Howard, R.A., Kreplin, R., Michels, D.J., Moses, J.D., Defise, J.M., Jamar, C., Rochus, P., Chauvineau, J.P., Marioge, J.P., Catura, R.C., Lemen, J.R., Shing, L., Stern, R.A., Gurman, J.B., Neupert, W.M., Maucherat, A., Clette, F., Cugnon, P., van Dessel, E.L.: 1995, EIT: Extreme-Ultraviolet Imaging Telescope for the SOHO Mission. Solar Phys. 162, 291. DOI. ADS.

Delannée, C., Artzner, G., Schmieder, B., Parenti, S.: 2014, Time Evolution of the Altitude of an Observed Coronal Wave. Solar Phys. 289, 2565. DOI. ADS.

Eselevich, V.G., Eselevich, M.V., Zimovets, I.V.: 2019, Observations of a Flare-Generated Blast Wave in a Pseudo Coronal Mass Ejection Event. Solar Phys. 294, 73. DOI. ADS.

Gao, G.-N., Wang, M., Lin, J., Wu, N., Tan, C.-M., Kliem, B., Su, Y.: 2014, Radio observations of the fine structure inside a post-CME current sheet. Research in Astronomy and Astrophysics 14, 843. DOI. ADS.

Gopalswamy, N., Yashiro, S., Temmer, M., Davila, J., Thompson, W.T., Jones, S., McAteer, R.T.J., Wuelser, J.-P., Freeland, S., Howard, R.A.: 2009, EUV Wave Reflection from a Coronal Hole. Astrophys. J. Lett. 691, L123. DOI. ADS.

Guo, Y., Ding, M.D., Chen, P.F.: 2015, Slow Patchy Extreme-ultraviolet Propagating Fronts Associated with Fast Coronal Magneto-acoustic Waves in Solar Eruptions. Astrophys. J. Sup. 219, 36. DOI. ADS.

Hu, H., Liu, Y.D., Zhu, B., Peter, H., He, W., Wang, R., Yang, Z.: 2019, Effects of Coronal Density and Magnetic Field Distributions on a Global Solar EUV Wave. Astrophys. J. $\mathbf{8 7 8}, 106$. DOI. ADS.

Hudson, H.S., Khan, J.I., Lemen, J.R., Nitta, N.V., Uchida, Y.: 2003, Soft X-ray observation of a large-scale coronal wave and its exciter. Solar Phys. 212, 121. DOI. ADS.

Karpen, J.T., Antiochos, S.K., DeVore, C.R.: 2012, The Mechanisms for the Onset and Explosive Eruption of Coronal Mass Ejections and Eruptive Flares. Astrophys. J. 760, 81. DOI. ADS.

Khan, J.I., Aurass, H.: 2002, X-ray observations of a large-scale solar coronal shock wave. Astron. Astrophys. 383, 1018. DOI. ADS.

Kliem, B., Török, T.: 2006, Torus Instability. 96, 255002. DOI. ADS.

Kozarev, K.A., Korreck, K.E., Lobzin, V.V., Weber, M.A., Schwadron, N.A.: 2011, Off-limb Solar Coronal Wavefronts from SDO/AIA Extreme-ultraviolet Observations-Implications for Particle Production. Astrophys. J. Lett. 733, L25. DOI. ADS.

Krause, G., Cécere, M., Zurbriggen, E., Costa, A., Francile, C., Elaskar, S.: 2018, Are CMEs capable of producing Moreton waves? A case study: the 2006 December 6 event. Mon. Not. Roy. Astron. Soc. 474, 770. DOI. ADS.

Kumar, P., Innes, D.E.: 2013, Multiwavelength Observations of an Eruptive Flare: Evidence for Blast Waves and Break-Out. Solar Phys. 288, 255. DOI. ADS.

Kumar, P., Innes, D.E.: 2015, Partial Reflection and Trapping of a Fast-mode Wave in Solar Coronal Arcade Loops. Astrophys. J. Lett. 803, L23. DOI. ADS.

Kumar, P., Manoharan, P.K.: 2013, Eruption of a plasma blob, associated M-class flare, and large-scale extreme-ultraviolet wave observed by SDO. Astron. Astrophys. 553, A109. DOI. ADS.

Kumar, P., Innes, D.E., Cho, K.-S.: 2016, Flare-generated Shock Wave Propagation through Solar Coronal Arcade Loops and an Associated Type II Radio Burst. Astrophys. J. 828, 28. DOI. ADS.

Kumar, P., Nakariakov, V.M., Cho, K.-S.: 2017, Quasi-periodic Radio Bursts Associated with Fast-mode Waves near a Magnetic Null Point. Astrophys. J. 844, 149. DOI. ADS. 
Kumar, P., Cho, K.-S., Chen, P.F., Bong, S.-C., Park, S.-H.: 2013, Multiwavelength Study of a Solar Eruption from AR NOAA 11112: II. Large-Scale Coronal Wave and Loop Oscillation. Solar Phys. 282, 523. DOI. ADS.

Lemen, J.R., Title, A.M., Akin, D.J., Boerner, P.F., Chou, C., Drake, J.F., Duncan, D.W., Edwards, C.G., Friedlaender, F.M., Heyman, G.F., Hurlburt, N.E., Katz, N.L., Kushner, G.D., Levay, M., Lindgren, R.W., Mathur, D.P., McFeaters, E.L., Mitchell, S., Rehse, R.A., Schrijver, C.J., Springer, L.A., Stern, R.A., Tarbell, T.D., Wuelser, J.-P., Wolfson, C.J., Yanari, C., Bookbinder, J.A., Cheimets, P.N., Caldwell, D., Deluca, E.E., Gates, R., Golub, L., Park, S., Podgorski, W.A., Bush, R.I., Scherrer, P.H., Gummin, M.A., Smith, P., Auker, G., Jerram, P., Pool, P., Soufli, R., Windt, D.L., Beardsley, S., Clapp, M., Lang, J., Waltham, N.: 2012, The Atmospheric Imaging Assembly (AIA) on the Solar Dynamics Observatory (SDO). Solar Phys. 275, 17. DOI. ADS.

Li, D., Ning, Z.J., Zhang, Q.M.: 2015, Imaging and Spectral Observations of Quasi-periodic Pulsations in a Solar Flare. Astrophys. J. 807, 72. DOI. ADS.

Li, D., Zhang, Q.M., Huang, Y., Ning, Z.J., Su, Y.N.: 2017, Quasi-periodic pulsations with periods that change depending on whether the pulsations have thermal or nonthermal components. Astron. Astrophys. 597, L4. DOI. ADS.

Li, T., Zhang, J., Yang, S., Liu, W.: 2012, SDO/AIA Observations of Secondary Waves Generated by Interaction of the 2011 June 7 Global EUV Wave with Solar Coronal Structures. Astrophys. J. 746, 13. DOI. ADS.

Liu, R., Liu, C., Xu, Y., Liu, W., Kliem, B., Wang, H.: 2013, Observation of a Moreton Wave and Wave-Filament Interactions Associated with the Renowned X9 Flare on 1990 May 24. Astrophys. J. 773, 166. DOI. ADS.

Liu, R., Wang, Y., Lee, J., Shen, C.: 2019, Impacts of EUV Wavefronts on Coronal Structures in Homologous Coronal Mass Ejections. Astrophys. J. 870, 15. DOI. ADS.

Liu, W., Ofman, L.: 2014, Advances in Observing Various Coronal EUV Waves in the SDO Era and Their Seismological Applications (Invited Review). Solar Phys. 289, 3233. DOI. ADS.

Liu, W., Title, A.M., Zhao, J., Ofman, L., Schrijver, C.J., Aschwanden, M.J., De Pontieu, B., Tarbell, T.D.: 2011, Direct Imaging of Quasi-periodic Fast Propagating Waves of $\sim 2000 \mathrm{~km}$ $\mathrm{s}^{-1}$ in the Low Solar Corona by the Solar Dynamics Observatory Atmospheric Imaging Assembly. Astrophys. J. Lett. 736, L13. DOI. ADS.

Liu, W., Ofman, L., Nitta, N.V., Aschwand en, M.J., Schrijver, C.J., Title, A.M., Tarbell, T.D.: 2012, Quasi-periodic Fast-mode Wave Trains within a Global EUV Wave and Sequential Transverse Oscillations Detected by SDO/AIA. Astrophys. J. 753, 52. DOI. ADS.

Liu, W., Ofman, L., Broder, B., Karlický, M., Downs, C.: 2016, Quasi-periodic fast-mode magnetosonic wave trains within coronal waveguides associated with flares and CMEs. In: American Institute of Physics Conference Series, American Institute of Physics Conference Series 1720, 040010. DOI. ADS.

Liu, W., Jin, M., Downs, C., Ofman, L., Cheung, M.C.M., Nitta, N.V.: 2018, A Truly Global Extreme Ultraviolet Wave from the SOL2017-09-10 X8.2+ Solar Flare-Coronal Mass Ejection. Astrophys. J. Lett. 864, L24. DOI. ADS.

Liu, Y.D., Zhu, B., Zhao, X.: 2019, Geometry, Kinematics, and Heliospheric Impact of a Large CME-driven Shock in 2017 September. Astrophys. J. 871, 8. DOI. ADS.

Long, D.M., DeLuca, E.E., Gallagher, P.T.: 2011, The Wave Properties of Coronal Bright Fronts Observed Using SDO/AIA. Astrophys. J. Lett. 741, L21. DOI. ADS.

Long, D.M., Bloomfield, D.S., Chen, P.F., Downs, C., Gallagher, P.T., Kwon, R.-Y., Vanninathan, K., Veronig, A.M., Vourlidas, A., Vršnak, B., Warmuth, A., Žic, T.: 2017, Understanding the Physical Nature of Coronal "EIT Waves". Solar Phys. 292, 7. DOI. ADS.

Ma, S., Raymond, J.C., Golub, L., Lin, J., Chen, H., Grigis, P., Testa, P., Long, D.: 2011, Observations and Interpretation of a Low Coronal Shock Wave Observed in the EUV by the SDO/AIA. Astrophys. J. 738, 160. DOI. ADS.

Magdalenić, J., Vršnak, B., Pohjolainen, S., Temmer, M., Aurass, H., Lehtinen, N.J.: 2008, A Flare-Generated Shock during a Coronal Mass Ejection on 24 December 1996. Solar Phys. 253, 305. DOI. ADS.

Magdalenić, J., Marqué, C., Zhukov, A.N., Vršnak, B., Veronig, A.: 2012, Flare-generated Type II Burst without Associated Coronal Mass Ejection. Astrophys. J. 746, 152. DOI. ADS.

Mann, G.: 1995, Simple magnetohydrodynamic waves. Journal of Plasma Physics 53, 109. DOI. ADS. 
Miao, Y.H., Liu, Y., Shen, Y.D., Li, H.B., Abidin, Z.Z., Elmhamdi, A., Kordi, A.S.: 2019, A Quasi-periodic Propagating Wave and Extreme-ultraviolet Waves Excited Simultaneously in a Solar Eruption Event. Astrophys. J. Lett. 871, L2. DOI. ADS.

Miao, Y., Li, D., Yuan, D., Jiang, C., Elmhamdi, A., Zhao, M., Anfinogentov, S.: 2021, Diagnosing a Solar Flaring Core with Bidirectional Quasi-periodic Fast Propagating Magnetoacoustic Waves. Astrophys. J. Lett. 908, L37. DOI. ADS.

Moore, R.L., Sterling, A.C., Hudson, H.S., Lemen, J.R.: 2001, Onset of the Magnetic Explosion in Solar Flares and Coronal Mass Ejections. Astrophys. J. 552, 833. DOI. ADS.

Moreton, G.E., Ramsey, H.E.: 1960, Recent Observations of Dynamical Phenomena Associated with Solar Flares. Pub. Astron. Soc. Pac. 72, 357. DOI. ADS.

Moses, D., Clette, F., Delaboudinière, J.-P., Artzner, G.E., Bougnet, M., Brunaud, J., Carabetian, C., Gabriel, A.H., Hochedez, J.F., Millier, F., Song, X.Y., Au, B., Dere, K.P., Howard, R.A., Kreplin, R., Michels, D.J., Defise, J.M., Jamar, C., Rochus, P., Chauvineau, J.P., Marioge, J.P., Catura, R.C., Lemen, J.R., Shing, L., Stern, R.A., Gurman, J.B., Neupert, W.M., Newmark, J., Thompson, B., Maucherat, A., Portier-Fozzani, F., Berghmans, D., Cugnon, P., van Dessel, E.L., Gabryl, J.R.: 1997, EIT Observations of the Extreme Ultraviolet Sun. Solar Phys. 175, 571. DOI. ADS.

Nakariakov, V.M., Verwichte, E.: 2005, Coronal Waves and Oscillations. Living Reviews in Solar Physics 2, 3. DOI. ADS.

Neupert, W.M.: 1968, Comparison of Solar X-Ray Line Emission with Microwave Emission during Flares. Astrophys. J. Lett. 153, L59. DOI. ADS.

Nisticò, G., Pascoe, D.J., Nakariakov, V.M.: 2014, Observation of a high-quality quasi-periodic rapidly propagating wave train using SDO/AIA. Astron. Astrophys. 569, A12. DOI. ADS.

Ofman, L.: 2007, Three-dimensional MHD Model of Wave Activity in a Coronal Active Region. Astrophys. J. 655, 1134. DOI. ADS.

Ofman, L., Thompson, B.J.: 2002, Interaction of EIT Waves with Coronal Active Regions. Astrophys. J. 574, 440. DOI. ADS.

Ofman, L., Liu, W., Title, A., Aschwanden, M.: 2011, Modeling Super-fast Magnetosonic Waves Observed by SDO in Active Region Funnels. Astrophys. J. Lett. 740, L33. DOI. ADS.

Olmedo, O., Vourlidas, A., Zhang, J., Cheng, X.: 2012, Secondary Waves and/or the "Reflection" from and "Transmission" through a Coronal Hole of an Extreme Ultraviolet Wave Associated with the 2011 February 15 X2.2 Flare Observed with SDO/AIA and STEREO/EUVI. Astrophys. J. 756, 143. DOI. ADS.

Pascoe, D.J., Nakariakov, V.M., Kupriyanova, E.G.: 2013, Fast magnetoacoustic wave trains in magnetic funnels of the solar corona. Astron. Astrophys. 560, A97. DOI. ADS.

Pesnell, W.D., Thompson, B.J., Chamberlin, P.C.: 2012, The Solar Dynamics Observatory (SDO). Solar Phys. 275, 3. DOI. ADS.

Priest, E.R.: 1982, Solar magneto-hydrodynamics. ADS.

Qu, Z.N., Jiang, L.Q., Chen, S.L.: 2017, Observations of a Fast-mode Magnetosonic Wave Propagating along a Curving Coronal Loop on 2011 November 11. Astrophys. J. 851, 41. DOI. ADS.

Reid, H.A.S., Ratcliffe, H.: 2014, A review of solar type III radio bursts. Research in Astronomy and Astrophysics 14, 773. DOI. ADS.

Roberts, B., Edwin, P.M., Benz, A.O.: 1983, Fast pulsations in the solar corona. Nature 305, 688. DOI. ADS.

Roberts, B., Edwin, P.M., Benz, A.O.: 1984, On coronal oscillations. Astrophys. J. 279, 857. DOI. ADS.

Schrijver, C.J., De Rosa, M.L.: 2003, Photospheric and heliospheric magnetic fields. Solar Phys. 212, 165. DOI. ADS.

Schrijver, C.J., Aschwanden, M.J., Title, A.M.: 2002, Transverse oscillations in coronal loops observed with TRACE I. An Overview of Events, Movies, and a Discussion of Common Properties and Required Conditions. Solar Phys. 206, 69. DOI. ADS.

Shen, Y., Liu, Y.: 2012a, Evidence for the Wave Nature of an Extreme Ultraviolet Wave Observed by the Atmospheric Imaging Assembly on Board the Solar Dynamics Observatory. Astrophys. J. 754, 7. DOI. ADS.

Shen, Y., Liu, Y.: 2012b, Observational Study of the Quasi-periodic Fast-propagating Magnetosonic Waves and the Associated Flare on 2011 May 30. Astrophys. J. 753, 53. DOI. ADS.

Shen, Y., Liu, Y.: 2012c, Simultaneous Observations of a Large-scale Wave Event in the Solar Atmosphere: From Photosphere to Corona. Astrophys. J. Lett. 752, L23. DOI. ADS. 
Shen, Y.-D., Liu, Y., Su, J.-T., Li, H., Zhang, X.-F., Tian, Z.-J., Zhao, R.-J., Elmhamdi, A.: 2013a, Observations of a Quasi-periodic, Fast-Propagating Magnetosonic Wave in Multiple Wavelengths and Its Interaction with Other Magnetic Structures. Solar Phys. 288, 585. DOI. ADS.

Shen, Y., Liu, Y., Su, J., Li, H., Zhao, R., Tian, Z., Ichimoto, K., Shibata, K.: 2013b, Diffraction, Refraction, and Reflection of an Extreme-ultraviolet Wave Observed during Its Interactions with Remote Active Regions. Astrophys. J. Lett. 773, L33. DOI. ADS.

Shen, Y., Ichimoto, K., Ishii, T.T., Tian, Z., Zhao, R., Shibata, K.: 2014, A Chain of Winking (Oscillating) Filaments Triggered by an Invisible Extreme-ultraviolet Wave. Astrophys. J. 786, 151. DOI. ADS.

Shen, Y., Liu, Y., Tian, Z., Qu, Z.: 2017a, On a Small-scale EUV Wave: The Driving Mechanism and the Associated Oscillating Filament. Astrophys. J. 851, 101. DOI. ADS.

Shen, Y., Liu, Y.D., Su, J., Qu, Z., Tian, Z.: 2017b, On a Solar Blowout Jet: Driving Mechanism and the Formation of Cool and Hot Components. Astrophys. J. 851, 67. DOI. ADS.

Shen, Y., Liu, Y., Song, T., Tian, Z.: 2018a, A Quasi-periodic Fast-propagating Magnetosonic Wave Associated with the Eruption of a Magnetic Flux Rope. Astrophys. J. 853, 1. DOI. ADS.

Shen, Y., Tang, Z., Li, H., Liu, Y.: 2018b, Coronal EUV, QFP, and kink waves simultaneously launched during the course of jet-loop interaction. Mon. Not. Roy. Astron. Soc. 480, L63. DOI. ADS.

Shen, Y., Tang, Z., Miao, Y., Su, J., Liu, Y.: 2018c, EUV Waves Driven by the Sudden Expansion of Transequatorial Loops Caused by Coronal Jets. Astrophys. J. Lett. 860, L8. DOI. ADS.

Shen, Y., Liu, Y., Liu, Y.D., Su, J., Tang, Z., Miao, Y.: 2018d, Homologous Large-amplitude Nonlinear Fast-mode Magnetosonic Waves Driven by Recurrent Coronal Jets. Astrophys. J. 861, 105. DOI. ADS.

Shen, Y., Chen, P.F., Liu, Y.D., Shibata, K., Tang, Z., Liu, Y.: 2019, First Unambiguous Imaging of Large-scale Quasi-periodic Extreme-ultraviolet Wave or Shock. Astrophys. J. 873, 22. DOI. ADS.

Shen, Y., Li, B., Chen, P., Zhou, X., Liu, Y.: 2020, Progress in Coronal EUV Research. Chin Sci Bull 65. DOI. http://engine.scichina.com/doi/10.1360/TB-2020-0748.

Su, W., Cheng, X., Ding, M.D., Chen, P.F., Sun, J.Q.: 2015, A Type II Radio Burst without a Coronal Mass Ejection. Astrophys. J. 804, 88. DOI. ADS.

Takasao, S., Shibata, K.: 2016, Above-the-loop-top Oscillation and Quasi-periodic Coronal Wave Generation in Solar Flares. Astrophys. J. 823, 150. DOI. ADS.

Thompson, B.J., Myers, D.C.: 2009, A Catalog of Coronal "EIT Wave" Transients. Astrophys. J. Sup. 183, 225. DOI. ADS.

Thompson, B.J., Plunkett, S.P., Gurman, J.B., Newmark, J.S., St. Cyr, O.C., Michels, D.J.: 1998, SOHO/EIT observations of an Earth-directed coronal mass ejection on May 12, 1997. Geophys. Res. Lett. 25, 2465. DOI. ADS.

Torrence, C., Compo, G.P.: 1998, A Practical Guide to Wavelet Analysis. Bulletin of the American Meteorological Society 79, 61. DOI. ADS.

Tripathi, D., Isobe, H., Jain, R.: 2009, Large Amplitude Oscillations in Prominences. Space Sci. Rev. 149, 283. DOI. ADS.

Uchida, Y.: 1968, Propagation of Hydromagnetic Disturbances in the Solar Corona and Moreton's Wave Phenomenon. Solar Phys. 4, 30. DOI. ADS.

Uchida, Y.: 1970, Diagnosis of Coronal Magnetic Structure by Flare-Associated Hydromagnetic Disturbances. Pub. Astron. Soc. Japan 22, 341. ADS.

Veronig, A.M., Muhr, N., Kienreich, I.W., Temmer, M., Vršnak, B.: 2010, First Observations of a Dome-shaped Large-scale Coronal Extreme-ultraviolet Wave. Astrophys. J. Lett. 716, L57. DOI. ADS.

Vršnak, B., Cliver, E.W.: 2008, Origin of Coronal Shock Waves. Invited Review. Solar Phys. 253, 215. DOI. ADS.

Wang, J., Yan, X., Kong, D., Xue, Z., Yang, L., Li, Q.: 2020, A Small-scale Filament Eruption Inducing a Moreton Wave, an EUV Wave, and a Coronal Mass Ejection. Astrophys. J. 894, 30. DOI. ADS.

Warmuth, A.: 2007, In: Klein, K.-L., MacKinnon, A.L. (eds.) Large-scale Waves and Shocks in the Solar Corona 725, 107. ADS.

Warmuth, A.: 2010, Large-scale waves in the solar corona: The continuing debate. Advances in Space Research 45, 527. DOI. ADS. 
Warmuth, A.: 2015, Large-scale Globally Propagating Coronal Waves. Living Reviews in Solar Physics 12, 3. DOI. ADS.

Warmuth, A., Vršnak, B., Magdalenić, J., Hanslmeier, A., Otruba, W.: 2004, A multiwavelength study of solar flare waves. II. Perturbation characteristics and physical interpretation. Astron. Astrophys. 418, 1117. DOI. ADS.

Wills-Davey, M.J., Thompson, B.J.: 1999, Observations of a Propagating Disturbance in TRACE. Solar Phys. 190, 467. DOI. ADS.

Xue, Z.K., Qu, Z.Q., Yan, X.L., Zhao, L., Ma, L.: 2013, Deformation and deceleration of coronal wave. Astron. Astrophys. 556, A152. DOI. ADS.

Yuan, D., Shen, Y., Liu, Y., Nakariakov, V.M., Tan, B., Huang, J.: 2013, Distinct propagating fast wave trains associated with flaring energy releases. Astron. Astrophys. 554, A144. DOI. ADS.

Zheng, R., Jiang, Y., Yang, J., Bi, Y., Hong, J., Yang, D., Yang, B.: 2012a, A Fast Propagating Extreme-Ultraviolet Wave Associated with a Mini-filament Eruption. Astrophys. J. 753, 112. DOI. ADS.

Zheng, R., Jiang, Y., Yang, J., Bi, Y., Hong, J., Yang, D., Yang, B.: 2012b, An Extreme Ultraviolet Wave Associated with a Micro-sigmoid Eruption. Astrophys. J. Lett. 753, L29. DOI. ADS.

Zheng, R., Jiang, Y., Yang, J., Bi, Y., Hong, J., Yang, B., Yang, D.: 2013, An Extremeultraviolet Wave Associated with a Surge. Astrophys. J. 764, 70. DOI. ADS.

Zhou, X., Liang, H.: 2017, The relationship between the 5-min oscillation and 3-min oscillations at the umbral/penumbral sunspot boundary. Astrophys. Space Sci. 362, 46. DOI. ADS.

Zhou, X., Liang, H., Li, Q., Zhang, W., OuYang, W.: 2017, Statistical research of the umbral and penumbral oscillations. New. Astron. 51, 86. DOI. ADS.

Zhukov, A.N., Auchère, F.: 2004, On the nature of EIT waves, EUV dimmings and their link to CMEs. Astron. Astrophys. 427, 705. DOI. ADS. 